\title{
Correction to: Multidomain Frailty in Heart Failure: Current Status and Future Perspectives
}

\author{
Shinya Tanaka ${ }^{1}$ (D) $\cdot$ Masashi Yamashita ${ }^{2}$ (D) $\cdot$ Hiroshi Saito ${ }^{3,4}$ (D) $\cdot$ Kentaro Kamiya $^{5}$ (D) Daichi Maeda $^{6} \cdot$ \\ Masaaki Konishi $^{7}$ - Yuya Matsue ${ }^{4,8}$ (D)
}

Published online: 10 May 2021

(C) Springer Science+Business Media, LLC, part of Springer Nature 2021

\section{Correction to: Current Heart Failure Reports https://doi.org/10.1007/s11897-021-00513-2}

Due to typesetting mistake, the reference numbers found in Fig. 1 were mismatched to other references.

The original article has been corrected.

Publisher's Note Springer Nature remains neutral with regard to jurisdictional claims in published maps and institutional affiliations.

The online version of the original article can be found at https://doi.org/ 10.1007/s11897-021-00513-2

Yuya Matsue

yuya8950@gmail.com

1 Department of Rehabilitation, Nagoya University Hospital, Aichi, Japan

2 Department of Rehabilitation Sciences, School of Medical Sciences, Kitasato University Graduate, Kanagawa, Japan

3 Department of Rehabilitation, KamedaMedical Center, Chiba, Japan

4 Department of Cardiovascular Biology and Medicine, Juntendo University Graduate School of Medicine, 2-1-1 Hongo, Bunkyo-ku, Tokyo 113-8421, Japan
Department of Rehabilitation, School of Allied Health Sciences, Kitasato University, Kanagawa, Japan

6 Department of Cardiology, Osaka Medical College, Osaka, Japan

7 Division of Cardiology, Yokohama City University Medical Center, Kanagawa, Japan

8 Cardiovascular Respiratory Sleep Medicine, Juntendo University Graduate School of Medicine, 2-1-1 Hongo, Bunkyo-ku, Tokyo 113-8421, Japan 